


\title{
Una aproximación sociolingüística al empleo del discurso referido en el corpus PRESEEA de Santiago de Chile*
}

\author{
A sociolinguistic approach of the reported speech use in the \\ PRESEE A corpus of Santiago, Chile
}

\author{
Abelardo San Martín \\ Universidad de Chile \\ Chile \\ asmartin@uchile.cl
}

\author{
Silvana Guerrero \\ Universidad de Chile \\ Chile \\ siguerrero@u.uchile.cl
}

Recibido: 13-VI-2012/ Aceptado: 27-XII-2012

\section{Resumen}

En este artículo se analiza el empleo del discurso referido en el habla de Santiago de Chile desde el punto de vista sociolingüístico. Para tal propósito, se estudiaron las secuencias discursivas en las que se manifestó dicho recurso en 54 entrevistas que forman parte del Corpus Sociolingüístico del PRESEEA en Santiago de Chile. Para el marco conceptual de esta investigación se consultaron los estudios sobre la polifonía del lenguaje (Ducrot, 1986; Reyes, 1993) y el análisis sociolingüístico del discurso (Silva-Corvalán, 2001; Serrano, 2006). En el análisis se consideró el discurso referido como un caso de variable sociolingüística, en un sentido amplio del concepto, con dos valores o variantes generales: el discurso directo y el discurso indirecto y con diferentes subtipos o variantes específicas de acuerdo con distintos niveles o grados de reformulación y distanciamiento del sujeto respecto de su enunciado. Consecuentemente, se correlacionaron los factores sociodemográficos de los sujetos con el empleo de dichas variantes, los que demostraron tener incidencia en su empleo. Por otra parte, como variable interna se analizó el tipo de secuencia discursiva en la que las variantes generales del discurso referido aparecían con mayor frecuencia, confirmando el predominio del empleo de dicho recurso en las narraciones, si bien su participación como parte del repertorio de recursos argumentativos de los sujetos también es importante. Finalmente, se verificó que las mujeres suelen emplear con mayor frecuencia el discurso directo como una estrategia pragmática mediante la cual intentan dotar de mayor teatralidad a sus narraciones en forma de 'mini-dramas'.

Palabras Clave: Sociolingüística, análisis del discurso, variación discursiva, español de Chile, discurso referido. 


\begin{abstract}
This article analyzes the reported speech use in Santiago de Chile from a sociolinguistic point of view. For this purpose, we studied the discursive sequences in which they are used in 54 interviews that form part of the Sociolinguistic Corpus PRESEEA in Santiago, Chile. Studies on the polyphony of language (Ducrot, 1986; Reyes, 1993) and sociolinguistic analysis of discourse (Silva-Corvalán, 2001; Serrano, 2006) were consulted for the conceptual framework of this research. On the one hand, the analysis considers the reported speech as a sociolinguistic variable case, in a broad sense of the concept, with two values or general forms: direct speech and indirect speech, and with different subtypes or specific variations according to different levels or degrees of reformulation and detachment of the subjects from the utterance. Consequently, socio-demographic factors of subjects were correlated with the use of these variants, which were shown to have a relative impact on their employment. On the other hand, as internal variable we analyzed the type of discourse in which the sequence general variants of reported speech appeared more frequently, confirming the predominant use of that resource in the narratives, but their participation as part of the repertoire of argumentative subjects is also important. Finally, it was found that women tend to use more frequently direct speech as a pragmatic strategy by which they intend to add, in form of 'mini-dramas', theatricality to their narratives
\end{abstract}

Key Words: Sociolinguistic, discourse analysis, discursive variation, Chilean Spanish, reported speech.

\title{
INTRODUCCIÓN
}

En este trabajo se realiza una aproximación sociolingüística en el marco del variacionismo al empleo del discurso referido en una muestra de 54 entrevistas que constituyen una parte del Corpus del Proyecto para el Estudio Sociolingüístico del Español de España y América (PRESEEA) correspondiente al habla de Santiago de Chile. El objetivo general de este estudio es analizar el comportamiento variable a nivel discursivo y la estratificación sociolingüística del empleo del discurso referido en el mencionado corpus de entrevistas. Nuestros objetivos específicos son, por un lado, describir las formas variantes mediante las cuales se realiza la función variable del discurso referido y, por otro, correlacionar su frecuencia de empleo tanto con el tipo de secuencia discursiva (narrativa o argumentativa) en la que se registra, como con las variables socio-demográficas edad, sexo y nivel sociocultural de los sujetos.

Una de las premisas de este estudio es que el comportamiento variable del discurso referido podría justificar su tratamiento en términos de una función variable a nivel discursivo con dos formas variantes o valores generales: discurso directo y discurso indirecto. Es bien conocida la controversia que ha suscitado la extensión del concepto analítico de variable sociolingüística a otros niveles lingüísticos que trasciendan el fonético ${ }^{1}$. A este respecto, consideramos que, la existencia de fenómenos discursivos como el que nos ocupa, parecieran apoyar la posición de quienes mantienen la capacidad heurística del concepto de variable sociolingüística más allá de los límites 
de la fonética, con las debidas precauciones y adaptaciones que amerite su aplicación en los niveles sintáctico, léxico o discursivo. Para tal efecto, el análisis se dividió en una fase cualitativa, en la que se identificaron las modalidades variantes -a nivel general y específico- de la función variable en estudio, y una fase cuantitativa, en la que se procedió a la correlación de la frecuencia de empleo de las variantes generales con el tipo de secuencia discursiva y las variables socio-demográficas antes señaladas. A este respecto, es necesario precisar que, por tratarse de un estudio aproximativo que solo considera la mitad del corpus PRESEEA-SA, hemos optado por no incluir ninguna prueba estadística. En consecuencia, en la sección de análisis y presentación de los resultados, se destacarán solo aquellas diferencias porcentuales evidentes y no se hará alusión a la significación estadística ni de la variable ni de las variantes generales y específicas del fenómeno en estudio.

\section{Marco teórico}

\subsection{Discurso referido: Definición y modalidades}

En lo fundamental, la conceptualización del fenómeno del discurso referido se ha ido configurando, entre otros aportes, con las reflexiones sobre las propiedades dialógicas de los textos de Bajtín (1985), la caracterización del aparato formal de la enunciación de Benveniste (1970), la polifonía de la enunciación desarrollada por Ducrot (1986) y el análisis de la intertextualidad de Kristeva (1986). Igualmente, son valiosos para la comprensión del fenómeno en el español los trabajos de Reyes (1984, 1990, 1993) y de Maldonado $(1991,1999)^{2}$. El discurso referido, en términos generales, es un recurso lingüístico que permite a los hablantes recrear una situación discursiva, lo que se materializa mediante la reproducción de los enunciados proferidos por las voces intervinientes en la situación evocada y la reconstrucción del correspondiente contexto de enunciación. De acuerdo con la supuesta fidelidad relativa de lo citado a lo originalmente proferido por las voces evocadas, el discurso referido puede adoptar ya sea la forma de discurso directo o discurso indirecto. En el discurso narrativo de corte literario suelen emplearse, además, los estilos de discurso referido que los teóricos de la literatura denominan estilo indirecto libre y discurso narrativizado. Con el propósito de alcanzar una perspectiva dinámica que integre la multiplicidad de variantes del recurso estudiado, en nuestro análisis, hemos adoptado una perspectiva amplia que considera el discurso referido como la función mediante la cual se evoca el discurso enunciado en otras situaciones de habla, incluyendo desde la reproducción de citas literales hasta la referencia general a otros actos de comunicación.

\section{1. 1. Discurso o estilo directo}

Desde el punto de vista formal, el discurso o estilo directo es un procedimiento mediante el cual quien habla (o escribe) incorpora a su discurso una secuencia textual, de procedencia propia o ajena, que es presentada como si supuestamente fuera reproducida de modo literal. En la lengua oral, aparte de las señales prosódicas, el empleo de un verbo introductor, de la clase de los verba dicendi, marca, por lo general, 
la porción de texto incorporado. El verbo introductor indica además la clase de acto de habla atribuida a lo citado. Desde el punto de vista de la función pragmática del estilo directo, se han indicado entre otras funciones las siguientes: la vivacidad, el dramatismo, la veracidad o la autenticidad, para el discurso narrativo y, si se trata de discurso argumentativo: la autoridad o la orientación argumentativa (Reyes, 1993; Maldonado, 1999).

\section{1. 2. Discurso o estilo indirecto}

Formalmente, el discurso o estilo indirecto se caracteriza por el empleo del 'que' complementizador -que actúa como introductor de las palabras citadas-, así como por la traslación tanto de los tiempos verbales como de las personas pronominales. Por otra parte, desde el punto de vista pragmático-discursivo, la adopción del discurso indirecto entraña una reformulación del discurso citado, que afecta a las expresiones referenciales y el sistema deíctico originalmente empleados, los que son reformulados de acuerdo con el punto de vista de quien habla. Según Reyes (1993), el estilo indirecto puede manifestarse, por lo menos: a) como estilo indirecto estándar, b) como estilo indirecto encubierto y, c) como estilo indirecto libre. Este último caso, la autora citada lo define como una técnica propia del discurso literario.

\section{1. 3. Discurso narrativizado}

El discurso narrativizado consiste en un tipo de relato de palabras que no implica una reproducción del discurso original (Genette, 1989), sino más bien una especie de resumen, en tanto que el sentido general del discurso referido sigue siendo el mismo. Por su parte, Perret (1994) plantea que el discurso narrativizado implica que hubo un locutor que emitió un discurso, pero que en el locutor actual aparece de manera totalmente integrado ('grado cero' del discurso referido). En el marco de la perspectiva amplia e integradora que hemos asumido en este estudio, hemos optado por considerar el discurso narrativizado como un tipo de discurso indirecto, debido a que también consiste en una especie de reformulación del discurso citado.

\subsection{El estudio de la variación discursiva y el concepto de variable sociolingüística}

En este estudio sugerimos la posibilidad de considerar el empleo del discurso referido como un caso de variable lingüística -en un sentido amplio del concepto-a nivel de discurso. Por lo tanto, un aspecto a tener en consideración es el problema de la extensión del ámbito de aplicación del concepto de variable sociolingüística más allá del plano fonético ${ }^{3}$. Como es sabido, en el marco del paradigma cuantitativo laboviano más clásico, una exigencia para la aplicación de este concepto era que las formas alternantes no conllevaran diferencias semánticas y, de esta manera, consistieran en dos o más formas diferentes de decir lo mismo (Labov, 1972). No obstante, con posterioridad a este debate, se ha defendido la necesidad de relativizar la exigencia de igualdad semántica de las variantes y reemplazarla por un criterio de comparabilidad o 
equivalencia funcional o pragmática (Lavandera, 1978; Silva-Corvalán, 2001; Serrano, 2007, 2011), que está en el centro de la fundamentación de este trabajo. En principio, según Moreno Fernández (1998: 19), la variación lingüística:

"definida como el uso alterno de formas diferentes de decir lo mismo, se puede encontrar prácticamente en todos los niveles de la lengua, desde el más concreto (fonético-fonológico) al más amplio (discurso, por ejemplo), pasando por la gramática y el léxico".

Por ello, la ampliación de los estudios variacionistas al plano discursivo constituye una transición lógica y necesaria. Un análisis cuantitativo del discurso referido es posible solo si se logra determinar que dos o más formas de este tipo resultan estar en 'distribución complementaria débil' (Sankoff, 1988) respecto de los factores sociodemográficos de los hablantes. En rigor, entonces, un análisis variacionista del discurso referido solo es posible en la medida en que se correlacionen dichos factores con formas que cumplen esta misma función en el discurso. Por lo tanto, previamente al análisis cuantitativo del discurso referido, es necesario que un análisis de tipo cualitativo identifique dos o más formas alternantes que comparten esta misma función en el discurso.

En nuestra opinión, la aplicación del concepto de variable sociolingüística al estudio del discurso referido es viable puesto que, precisamente, las variantes de este recurso cumplen una misma función a nivel pragmático, esto es, referir o reproducir el discurso propio o ajeno enunciado en otras situaciones de habla. En este sentido, las variantes de discurso referido satisfarían el requisito de ser variantes pragmáticamente equivalentes; equivalencia tantas veces anhelada en el estudio de la variación sintáctica. El meollo del problema radica, más bien, en identificar con precisión la función discursiva 'variable' y sus correspondientes formas 'variantes' en una muestra representativa y estratificada de sujetos de una determinada comunidad de habla. Por lo que atañe al estudio de la variación discursiva en el habla de Santiago de Chile, en general, la perspectiva adoptada en el presente artículo ha sido ya aplicada con éxito en trabajos sobre las diferencias de género en el empleo del discurso referido en el estrato bajo (Prieto \& San Martín, 2002-2003), acerca de las diferencias de género en el componente evaluativo y la estructuración de patrones narrativos en el habla juvenil (Guerrero 2011a, 2011b), y sobre algunos tipos de marcadores del discurso como los reformuladores de distanciamiento y los marcadores de control de contacto (San Martín, 2004-2005, 2011, respectivamente).

\section{Metodología}

\subsection{Corpus}

El corpus que sirvió de base para el análisis del presente estudio consistió en 54 entrevistas sociolingüísticas que corresponden a la mitad del corpus de Santiago de Chile en el Proyecto para el Estudio Sociolingüístico del Español de 
España y América (PRESEEA)4. Para la recopilación del corpus sociolingüístico de PRESEEA en Santiago de Chile, se aprovechó, en lo principal, la instancia de aplicación de un instrumento que, para fines de la investigación sociolingüística del habla de la mencionada ciudad, se realiza anualmente desde 1978 hasta la fecha, en el marco de la asignatura de Sociolingüística que se imparte en las Licenciaturas en Lengua y Literatura Hispánica y Lengua y Literatura Inglesas de la Universidad de Chile ${ }^{5}$. Las entrevistas fueron hechas a hombres y mujeres con características sociodemográficas congruentes, aplicando los supuestos metodológicos sugeridos por Labov (1972). Consecuentemente, en dichas situaciones los entrevistadores debían tratar de superar la 'paradoja del observador' (Labov, 1972) consiguiendo, de esta forma, una muestra significativa de discurso natural grabado (estilo vernacular) de hablantes representativos de la comunidad de habla en estudio. En las instrucciones a los entrevistadores, se enfatizó que durante la entrevista debía generarse un clima de confianza, a fin de obtener el estilo de habla más espontáneo o natural del sujeto. Asimismo, se les indicó que, idealmente, los hablantes debían olvidarse de que estaban siendo grabados y debían comportarse de modo espontáneo como si estuvieran participando en una conversación natural.

\subsubsection{Población de la muestra}

Para la conformación de la muestra de sujetos entrevistados se consideró la población santiaguina que cumpliera con los siguientes criterios de asignación de hablante nativo de Santiago de Chile: 1) haber nacido y residido en forma ininterrumpida en Santiago, 2) haber residido en forma ininterrumpida en Santiago desde los cinco años de edad y 3) haber nacido en Santiago y haber residido en Santiago la mayor parte de sus vidas, salvo por periodos que sumados no superen los cuatro años en el tramo de 50 años y más y los tres años en el tramo de 35 a 49 años (Prieto, 1995-1996). El cuestionario de la entrevista se aplicó a una muestra de sujetos del tipo denominado 'muestra por cuotas con afijación uniforme', en la que se divide a la población en estratos o categorías y se asigna una cuota a cada uno de los distintos estratos. La muestra así conformada comprende un total de 54 individuos, distribuidos proporcionalmente como se indica en la Tabla 1 que se incluye a continuación.

Tabla 1. Distribución de sujetos de la muestra por nivel educacional, sexo y edad, según PRESEEA.

\begin{tabular}{|l|c|c|c|c|c|c|c|}
\hline \multicolumn{7}{|c|}{ Grupo de edad } \\
\cline { 1 - 8 } Nivel educacional & \multicolumn{2}{|c|}{$20-34$} & \multicolumn{2}{c|}{$35-54$} & \multicolumn{2}{c|}{55 y más } & \multirow{2}{*}{ Totales } \\
\cline { 2 - 8 } & $\mathrm{H}$ & $\mathrm{M}$ & $\mathrm{H}$ & $\mathrm{M}$ & $\mathrm{H}$ & $\mathrm{M}$ & \\
\hline Alto & 3 & 3 & 3 & 3 & 3 & 3 & 18 \\
\hline Medio & 3 & 3 & 3 & 3 & 3 & 3 & 18 \\
\hline Bajo & 3 & 3 & 3 & 3 & 3 & 3 & 18 \\
\hline Totales & 9 & 9 & 9 & 9 & 9 & 9 & 54 \\
\hline
\end{tabular}




\subsubsection{Procedimiento de estratificación empleado}

Siguiendo el sistema de estratificación del Proyecto PRESEEA, se estratificó a la muestra de sujetos de acuerdo con sus respectivos niveles de instrucción, es decir, según la equivalencia entre los niveles de estudios básico, secundario y superior con los estratos sociales bajo, medio y alto, respectivamente. Sin embargo, con el propósito de garantizar la homogeneidad en la composición de los distintos estratos de la muestra y, por tanto, la representatividad de los mismos en relación con la población analizada, se complementó dicho sistema de estratificación con el procedimiento de estratificación empleado por el proyecto de Estudio Sociolingüístico del Español de Chile (ESECH) ${ }^{6}$, que se basa en el procedimiento sugerido por Prieto (1995-1996). Dicho sistema de estratificación considera una escala de estatus socioeconómico que contempla las siguientes variables independientes: nivel educacional, categoría ocupacional y calidad ambiental de la zona de residencia. Asimismo, para asegurar la rigurosidad de la clasificación socioeconómica de los integrantes de la muestra, se tuvo en cuenta la estratificación social elaborada en 2008 por la Asociación chilena de empresas de Investigación de Mercado (AIM), la descripción básica de grupos socio-económicos realizada por la empresa ICCOM en 2007, así como la información específica proporcionada en 2003 por la empresa ADIMARK. De este modo, se escogieron las entrevistas realizadas a aquellos sujetos que, tras la aplicación de la escala de estratificación social, mostraban lo que Lenski $(1954,1956)$ denominó 'cristalización o congruencia de estatus'?

\section{Presentación y análisis de los resultados}

\subsection{Análisis cualitativo del discurso referido empleado en PRESEEA-SA}

A partir de la revisión de 18 entrevistas de la muestra correspondientes a una por casilla de la Tabla 1, se verificó que, para el cumplimiento de la función pragmáticodiscursiva de hacer referencia al o reproducir el discurso propio o ajeno enunciado en otras situaciones de habla, los sujetos cuentan con una variedad de recursos, de los que se pueden desprender, a lo menos, tres variantes específicas de discurso directo y dos de discurso indirecto, según la siguiente descripción ${ }^{8}$ :

1. Discurso Directo Libre (DDL): Reproducción aproximativa a lo literal del discurso propio o de otros interlocutores emitido en situaciones de enunciación diferentes a la actual sin presencia de elementos marcadores o introductores como pronombres personales o verbos de habla (verba dicendi). Se trata de la mera yuxtaposición de la cita sin marcas de tipo gramatical o léxico, sólo prosódicas.

Ejemplo: y la cuestión es que/ "tía ¿sabe? me penaron" / y toda la cuestión (SCHI_H11_001). 
2. Discurso Directo con Pronombre Personal (DDPRO): Reproducción aproximativa a lo literal del discurso propio o de otros interlocutores emitido en situaciones de enunciación diferentes a la actual utilizando los pronombres personales como elementos marcadores o introductores. En este caso, un pronombre personal funciona como introductor de la cita.

Ejemplo: (...) y me empezaban a meter conversa y yo "i¿quién es esta huevona?!” (SCHI_M13_074).

3. Discurso Directo Convencional (DDC): Reproducción aproximativa a lo literal del discurso propio o de otros interlocutores emitido en situaciones de enunciación diferentes a la actual utilizando los verbos de habla (verba dicendi) como elementos marcadores o introductores. Se trata del discurso directo convencional en el que un verbo de comunicación introduce la cita y las expresiones referenciales y el sistema deíctico de la situación original se mantienen.

Ejemplo: y yo dije: “yo quiero salir” (SCHI_M12_045).

4. Discurso Indirecto Convencional (DIC): Reformulación del discurso propio o de otros interlocutores emitido en situaciones de enunciación diferentes a la actual. El empleo de los verbos de habla (verba dicendi) y del elemento que (complementizador) con la función de introductor del discurso citado (complemento) son obligatorios. Se trata del discurso indirecto convencional, en el que las expresiones referenciales y el sistema deíctico de la situación original se adecúan al momento de habla actual. La reformulación opera sustancialmente a nivel gramatical.

Ejemplo: Y me dijo que él después me iba a dejar acá (SCHI_H22_049).

5. Discurso Indirecto Narrativizado (DIN): Referencia simple a un acto de discurso propio o de otros interlocutores emitido en situaciones de enunciación diferentes a la actual. La referencia implica la utilización de un verbo o de una expresión verbal que transmite de modo más o menos transparente la intención comunicativa y el contenido del acto de habla referido. En este caso, un verbo de comunicación funciona como elemento sintetizador de la cita. La reformulación opera sustancialmente a nivel semántico.

Ejemplo: y yo le conté lo que realmente estaba estudiando (SCHI_H11_002).

Es importante señalar que el discurso directo aparece mencionado como uno de los rasgos caracterizadores de la lengua oral en Chafe (1979, 1980, 1982). En su caracterización de las diferencias de la dicotomía oralidad/escritura, dicho autor propone los siguientes indicadores: implicación (involvement), distanciamiento (detachment), fragmentación e integración. Así, en su esquema, el discurso directo sería un rasgo del indicador de implicación (personal del enunciador en su discurso), mientras que el estilo indirecto sería un rasgo del indicador de distanciamiento? . Las 
variantes antes descritas se vincularon con su grado de reformulación gramatical y con el efecto pragmático de la secuencia, en términos de la relativa vivacidad o realismo de la cita, así como con las categorías de implicación (involvement) y distanciamiento (detachment) propuestas por Chafe $(1979,1980,1982)$. De este modo, se estableció un continuo o escala de discurso referido que va desde el menor nivel de reformulación y distanciamiento y mayor grado de realismo e implicación, que determina el empleo del Discurso Directo Libre (DDL), hasta el mayor nivel de reformulación y distanciamiento y menor grado de realismo e implicación, característico del empleo del Discurso Indirecto Narrativizado (DIN). En la Tabla 2 se presenta e ilustra la matriz de análisis empleada en el procesamiento de la muestra:

Tabla 2. Matriz de análisis.

\begin{tabular}{|l|}
\hline \multicolumn{1}{|c|}{ Tipificación de fenómenos } \\
\hline \multicolumn{1}{|c|}{ A. Discurso Directo (DD) } \\
\hline \multicolumn{1}{|c|}{ + Directo: - reformulación + realismo + implicación - distanciamiento } \\
\hline $\begin{array}{l}\text { 1. Discurso Directo Libre (DDL) } \\
\text { entonces "No tienes por qué hacer eso... no te metas" }\end{array}$ \\
\hline $\begin{array}{l}\text { 2. Discurso Directo con Pronombre Personal (DDPRO) } \\
\text { Y entonces yo: "No tienes por qué hacer eso... no te metas" }\end{array}$ \\
\hline $\begin{array}{l}\text { 3. Discurso Directo Convencional (DDC) } \\
\text { Y entonces yo le dije: "No tienes por qué hacer eso... no te metas" }\end{array}$ \\
\hline \multicolumn{1}{|c|}{ B. Discurso Indirecto (DI) } \\
\hline $\begin{array}{l}\text { Y Discurso Indirecto Convencional (DIC) } \\
\text { 5. Discurso Indirecto Narrativizado (DIN) } \\
\text { Y entonces lo reté por metido }\end{array}$ \\
\hline - Directo: + reformulación - realismo - implicación + distanciamiento \\
\hline
\end{tabular}

Como puede apreciarse, en términos de Searle (1969), en todas estas modalidades variantes se mantiene constante la misma fuerza ilocucionaria (intencionalidad), esto es, referir otras situaciones de enunciación; lo que se modifica son los efectos perlocucionarios dependiendo del punto en que cada una se encuentre en la escala. En nuestra opinión, la escala de discurso referido antes señalada, en tanto describe formas alternativas de evocar otras situaciones de comunicación, puede encontrar una interpretación adecuada si la vinculamos con la noción de 'estilo discursivo', en particular, siguiendo las directrices analíticas propuestas por Coupland (2007) para quien el estilo es una forma de hacer algo. En este sentido, para Aijón Oliva y Serrano (2010: 4), por ejemplo:

"el estilo lingüístico [...] puede entenderse como cualquier forma de comunicarse que suponga una elección entre otras posibles, en un contexto interactivo determinado [...] es también, y muy especialmente, una manera de construir y comunicar significados". 
Asimismo, Eckert y Rickford (2001) se refieren al estilo como una dimensión dinámica que permite la construcción de la identidad individual y que interactúa tanto con contenidos específicos, como con factores contextuales que producen los significados.

Otro argumento a favor de esta interpretación es que revitaliza la denominación tradicional que ya desde la teoría literaria más clásica reservaba el término 'estilo' para estos recursos evocadores. Por consiguiente, dentro del discurso referido podrían considerarse formas alternantes que serían el resultado de estrategias estilísticas diferentes: un estilo de discurso más directo (es decir, menos reformulado, más realista, más implicado y menos distanciado), por una parte, y un estilo de discurso más indirecto (esto es, más reformulado, menos realista, menos implicado y más distanciado), por otra.

\subsection{Análisis cuantitativo del empleo de discurso referido en PRESEEA-SA}

En las secciones siguientes, se presentará el análisis cuantitativo de las secuencias discursivas en las que se manifestó el discurso referido en una muestra de 54 entrevistas que conforman el Corpus Sociolingüístico del PRESEEA en Santiago de Chile. A partir de la revisión de dichas entrevistas, se identificaron 1.095 casos en los que los sujetos cumplieron la función de referir el discurso propio o ajeno enunciado en otras situaciones de habla. Cabe destacar que, debido a la naturaleza aproximativa de nuestro estudio, hemos optado por recurrir a procedimientos estadísticos más simples (porcentajes de frecuencia).

En el Gráfico 1 se presentan las frecuencias de empleo de las variantes generales del discurso referido registradas en el corpus, esto es, discurso directo (DD) y discurso indirecto (DI).

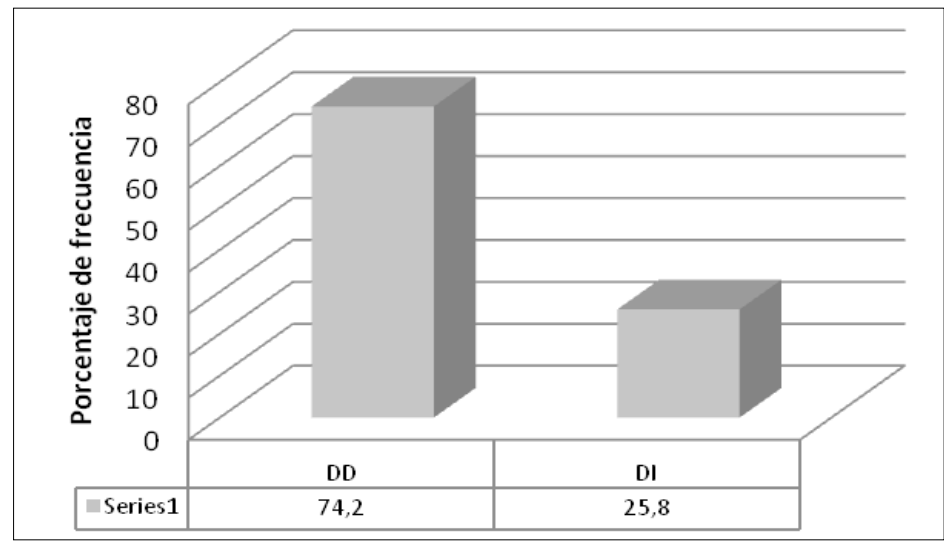

Gráfico 1. Frecuencias de variantes generales de discurso referido en PRESEEA-SA. 
Como se observa en el Gráfico 1, en el corpus PRESEEA-SA, se registró una mayor frecuencia de DD correspondiente al 74,2\% (813/1.095), en comparación a la ocurrencia de DI que solo constituyó el 25,8\% (282/1.095) del total de la muestra. Por lo que refiere a la frecuencia de empleo registrada por cada una de las variantes específicas de discurso referido, en el Gráfico 2, se muestra la estadística correspondiente.

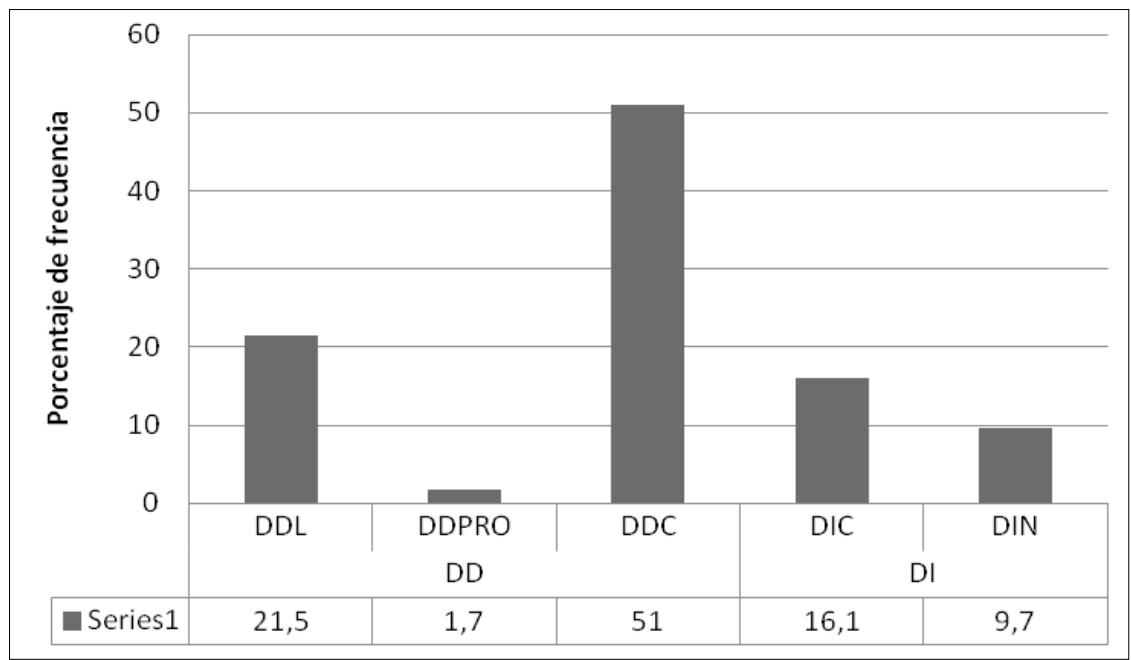

Gráfico 2. Frecuencia de las variantes específicas de discurso referido en PRESEA-SA.

Como se desprende del Gráfico 2, la mayor frecuencia de empleo de variantes específicas de discurso referido se registró en la utilización del DDC con el 51\% (559/1.095) de las ocurrencias. Le sigue, en orden descendente, el DDL correspondiente al 21,5\% (235/1.095), el DIC empleado el 16,1\% (176/1.095), el DIN con el 9,7\% (106/1.095) y, por último, el DDPRO con apenas el 1,7\% (19/1.095). En lo que sigue, se presentan algunos ejemplos ilustradores de cada recurso.

a) Ejemplos de variantes de DD:

(1) I: adentro [d]el baño y $\mathrm{L}$ me dijo no la recibai/ qué vai a recibir esa palomera (DDC) / eh y como yo era la que ehtaba inhcrita llegué / entré / me dijo ¿acepta la casa uhté o no la acepta / no la acepto (DDC) le [d] ije yo / me dijo pero eh que el sueldo de su mari[d]o eh pa[ra] que tenga una casa mejor (DDC)/y qué sacaba con el sueldo de mi mari[d]o si no me daba / entonceh dije no no no no (DDC) le dije yo // yo acepto (DDL) / así que me puse a firmar papeleh mierda, como mala [d]e la ca[b]eza y él afuera en la calle poh/ [es]taba la/ allí en Caracah la oficina /y dehpuéh me dijo ela aceptahte? o sea ¿la rechazahte? (DDC)/ no / la acepté / y si querí te vai con nosotroh / o si no búhcate donde irte / pero yo mi casa la la voy a 
tener como sea a como dé lugar (DDL)/ y ahí me vine poh con a ver ehta[b] a J, la M, la Ch y J / J ehtaba chiquitito (SCHI_M31_032).

(2) I: bueno / carrete carrete normal /ya / me llama el N / oye ya voy a hacer un carrete en mi casa (DDL)/ y yo le dije yo le digo ya voy a estar allá como las diez llego (DDC)/ ee bueno ahora no ehtoy tomando tanto pero su roncito loco / partiendo hablando de la vi[d]a / despuéh llega máh gente / y gente que no veíh hace tiempo / hola cómo estai (DDL)/ sale la guitarra / y empiezan a tocar temah tema y tema y / bueno/ en realida[d] / son como carreteh largoh / pero ahora no son tan así como como / como reventa[d]oh / son carreteh largoh porque tocamoh música y entreteni[d]o / duran hahta como lah siete /por ahí / ee la[s] buena[s] conversacion[es] / claro / claro como te dije de la buena conversación siempre salen amihtadeh / entonces yo creo que por eso tengo que mih mejoreh amigoh son los que he conversa[d]o más (SCHI_H13_075).

b) Ejemplo de variantes de DI:

(3) I: au / hay algunah que se pueden contar y otras que no se pueden contar poh /uuuh Dios mío/ a ver / plancha / plancha / ah por ejemplo en una oportunidad /en una oportunidad / por un asunto del colegio / hace poco fue eso / convocaron a una reunión / ehte colegio es de una fundación entonceh convocaron a una reunión y me llama una secretaria de la fundación y me dice de que < sic> están una reunión la directiva en tal parte y por qué yo no he ido si yo debería estar ahi (DIC) / entonceh yo rápidamente me fui a la reunión/ llegué/ ya estaban todoh reunidoh hacía ya hacía mucho rato/ incluso pedí permiso (DIN) y pasé a ocupar uno de los puestoh que estaban desocupadoh más adelante / y ya poh estuve en la reunión que duró alrededor de doh horah doh horah y media / y cuando terminó la reunión yo me di cuenta que me había metido a la reunión equivocada / no era la reunión que yo tenía que estar/ esa era otra comisión y yo me había equivocado / ahí me dio mucha plancha / pero como yo soy muy locuaz no tengo problemah de socialización o de manejo del lenguaje aparentemente / les dije creo que he venido a la reunión equivocada (DDC) y más encima yo habia opinado habia dado ideas / sugerencias / habia preguntado (DIN) había sido súper protagonista en esa reunión / pero nadie se enojó / todos se rieron y yo aprendí mucho (SCHI_H23_086).

\subsection{Análisis del empleo de las variantes generales del discurso referido según el tipo de secuencia discursiva}

Para el análisis correlacional de las secciones que siguen, hemos optado por presentar los resultados de las variantes generales, ya que las diferencias porcentuales de las variantes específicas resultaron ser irrelevantes según el tipo de análisis realizado. De acuerdo con nuestro análisis, de los 1.095 casos de ocurrencia del discurso referido, 
en 1.063 casos se lo empleó en secuencias narrativas o argumentativas de discurso, y en los 32 casos restantes en secuencias discursivas de otro tipo que, por lo tanto, no fueron consideradas en el análisis porcentual de esta sección. A continuación, en el Gráfico 3, se presentan los registros de frecuencia, según el tipo de secuencia discursiva, narrativa o argumentativa, en la que se observó el empleo de las variantes generales de discurso referido.

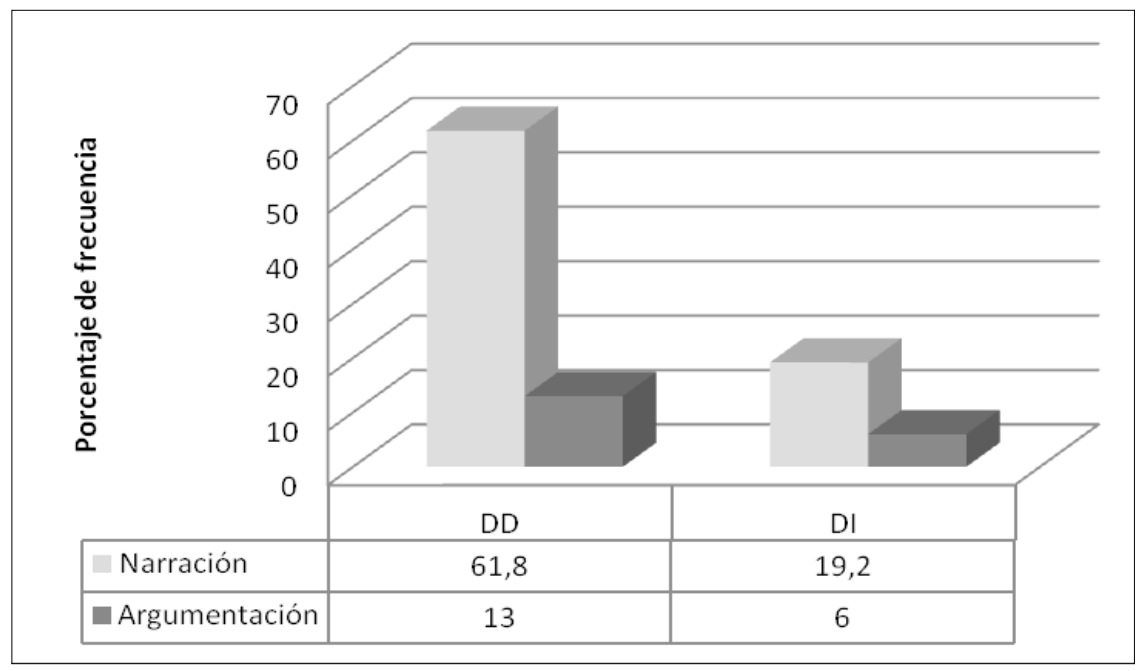

Gráfico 3. Frecuencia de las variantes generales de discurso referido según tipos de secuencia discursiva.

De acuerdo con los datos proporcionados en el Gráfico 3, tanto el DD como el DI se registran mayoritariamente en las secuencias discursivas de tipo narrativo, aunque con una evidente predominancia del DD. En concreto, el 61,8\% (657/1.063) de los casos en que se manifestó el DD corresponde a narraciones, en comparación con el empleo del mismo recurso registrado en las secuencias argumentativas, que apenas constituye un 13\% (138/1.063). Por lo que refiere al comportamiento del DI, en las secuencias narrativas, se manifestó en un 19,2\% (204/1.063) del total de la muestra y, en mucho menor proporción, se registró en las secuencias argumentativas, con tan solo un $6 \%$ (64/1.063) del total de casos. A continuación, ilustramos el empleo del discurso referido en ambos tipos de secuencia discursiva.

c) Ejemplo de discurso referido en secuencia discursiva de tipo narrativo:

(4) I: con metralleta hue[v]ón/ si no era na[da]/ no eran ni armamento yo/a [v] er yo me acuerdo ha[b]er vihto doh metralletah/ y doh armah cortah que no sé qué serían/ si eran doh y doh/ y me meten en la maleta/ y yo que[d]é sangrando y dehpué[s]/ y ahí se su[b]ieron/ me/ me cerraron la/ la maleta// y empezaron ahí a manejar poh// [en]tonceh en eso yo iba pensando qué es lo que hago (DDC)/ aunque en estah situacioneh así yo no nunca he senti[d] 
o muy miedo ah mucho miedo/ no no no no no// má[s] o menoh tranquilo en eso// entonceh yo me fui pensando y dije/ como yo la maleta la sabía abrir por dentro/ dije pue[d]o abrir la maleta y que pero tengo que esperar el momento (DDC) /E: claro/ I: que ellos paren/ de que yo vea vehículoh (DDC) porque no sabía dónde iban/ que yo vea ruedah// y cuando partan tirarme porque si dihparan la (DDC) / E: claro / I: me llega (DDC)// y así fui poh/ abrí la maleta por dentro y fui mirando y no veía nada y se dieron cuenta en una que iba la maleta abierta// pero pensaron que a elloh le había queda[d]o mal cerra[d]a y pararon y me la cerraron/ [en]tonce[s] yo dije tengo que tener más precaución (DDC)/la abro /E: sí/ I: la abro pero la voy afirmando con máh cuida[d]o// se dieron unah vueltah// y en una veo a u/ veo ruedah de vehículo// dije jesta! (DDC) / E: le[v]antabáih un poquito pa[ra] poder ver / I: vi nada/ vi rue[d]as// y se notaba que había un semáforo porque pararon// [en]tonceh dije esta es la mía/ de aquí a yo gano segundoh así que elloh parten pero una veh que partan yo me tiro/ ellos van a tener que cruzar la calle y yo voy a arrancar en contra (DDC) (SCHI_H22_051).

d) Ejemplo de discurso referido en secuencia discursiva de tipo argumentativo:

(5) E: ¿por qué te gusta ir a a Buenos Aireh? / I: porque me encanta la vida que hay en Buenos Aireh /ponte tú /si a la una de la mañana decíh tengo ganah de hacer esto (DDC) no eh como acá que es tengo ganah de hacer ehto / bueno mañana lo haré (DDL)/porque está todo cerrado acá / allá está siempre todo abierto hay / hay movimiento hay hay cultura que acá no hay / y no eh cara / no eh barata pero no eh cara (SCHI_M13_080).

\subsection{Análisis del empleo del discurso referido según variables sociodemográficas}

En el Gráfico 4, se presenta la distribución de frecuencias de empleo de las variantes generales del discurso referido según la variable sexo de los informantes. 


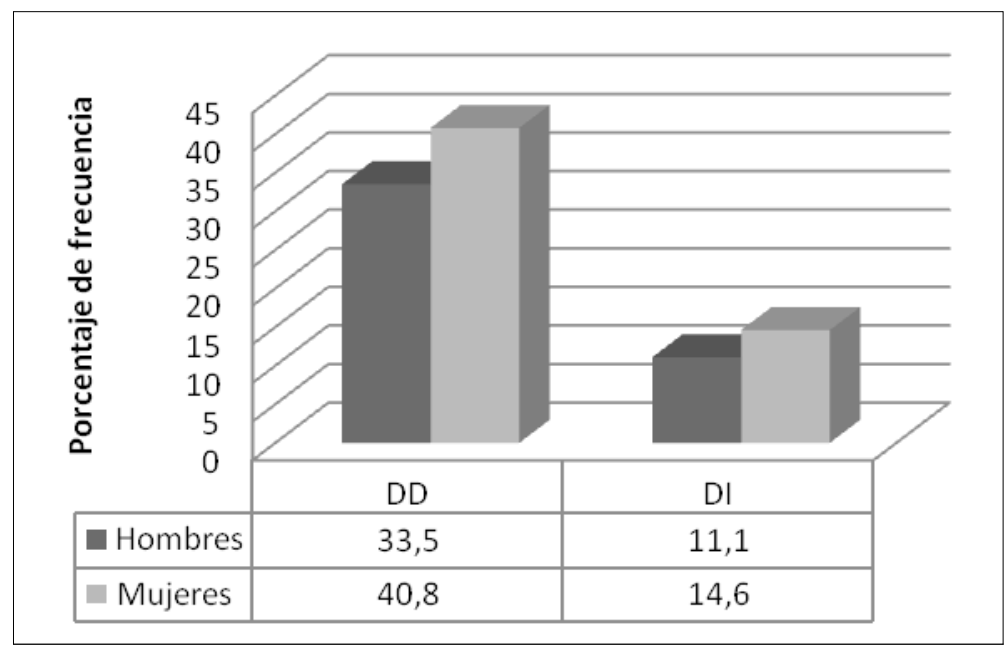

Gráfico 4. Variantes generales de discurso referido según sexo.

A partir del Gráfico 4, se puede destacar, en primer lugar, que el DD, tanto en hombres como en mujeres mostró una amplia aparición en comparación con los casos en los que se registró el DI en ambos sexos. En segunda instancia, observamos que el empleo del discurso referido, como variable, en el corpus, se manifestó preferentemente en las mujeres, aunque se trata de frecuencias de aparición muy cercanas a las registradas en las entrevistas de los hombres que conforman la muestra. En concreto, las mujeres utilizan el DD en el 40,8\% (447/1.095) de las ocurrencias, mientras que los hombres lo emplean en el 33,5\% (366/1.095). Por su lado, el DI se registró el 14,6\% (160/1.095) en entrevistas de mujeres, en comparación con los hombres, que lo utilizaron el 11,1\% (122/1.095) del total de la muestra.

Pese a que desde el punto de vista cuantitativo, en términos de frecuencias porcentuales generales se registraron frecuencias de empleo de las variantes generales del discurso referido muy cercanas entre hombres y mujeres (en el DD, 33,5\% los hombres y 40,8\% las mujeres, y en el DI, 11,1\% los hombres y 14,6\% las mujeres), una aproximación más interpretativa del fenómeno develó que las mujeres suelen emplear el DD como una estrategia pragmática mediante la cual intentan dotar de mayor teatralidad a sus narraciones en la forma de 'mini-dramas'. Es decir, a diferencia de lo que ocurre en las narraciones producidas por los hombres que conforman la muestra, en los relatos femeninos existe una tendencia a la proliferación del mencionado recurso en un mismo intercambio, intercalándose una gran cantidad de voces en el discurso, como se observa en el ejemplo 6.

(6) I: pero cuando yo me iba acercando para bajar / obvio / me paré poh y en ese momento subieron esoh do treh personah / y yo traía mi mi cartera atravesa[d] a aquí / pero en mi guatita voy yo así // y yo sentí cuando la muj me metió la 
mano aquí la mujer / le sentí la mano // entonceh sobre la mihma/ me veo y no la tengo / ¡mi chauchera! (DDL) me habían sacado mi chauchera / imagínese le ehtoy hablando de casi diez añoh atráh / yo traía algo de // parece que ocho mil pesoh // entonceh le vengo // yo // así con ehtah palabrah // le digo yo me (DDC)/ ¡ah! entonceh me dice el chofer ¡ay! ¿se va a bajar señora? (DDC) / no (DDC) le dije yo me bajo en loh carabineroh (DDC) mira / yo no sé de a dónde saqué fuerzah no (DDC) le dije yo me bajo en loh carabineroh (DDC) le dije yo / le dije y le digo a la mujer así y la la liebre llena / le dije ¡uhted! le digo y así la la la indico uhted ¿me di / me puede entregar le dije yo mi chauchera? que uhted me la sacó de acá (DDC)// y se hizo la lesa ¿qué? (DDC) me dijo ¡sí! le dije uhted / sí uhted fue (DDC) le dije porque yo la vi cuando la sacó / la sentí (DDC) entonceh me dice ¡no! (DDC) me dice ¡cómo se le ocurre! (DDC) y viene viene ¡ah! y uno se había queda[d]o en la pisadera // no subió // entonceh me dice ¡ay señora! (DDC) me dijo bueno (DDC) le dije iya! (DDC) le dije igual / que me iba a bajar en loh carabineroh (DDC) le dije yo / anteh no me bajo (DDL)// entonceh viene / el que ehtá en la pisadera señora me dice tiene que haber si[d]o el que se bajó (DDC) me dice / unoh que se bajaron ahí (DDL) / no (DDC) le dije yo / no fueron elloh fue uhted (DDL) // claro como yo le / yo me no sé qué cara me puse de enoja[d]a / le dije ¡no! si no me voy a bajar (DDC) / señora me dice mire pa[ra] pa[ra] abajo (DDC) me dice / ahí ehtá su chauchera en el suelo (DDL) // yo la recojo // yo le dije entre mí / mire lo que pensé / no importa dije si me ha sacado la plata pero pa[ra] que vea // de que así no máh no me la va a llevar (DDC)/ o sea que me di cuenta / tomé el chauchero claro / y juhto si me tocaba bajarme ya poh / me bajo // y cuando me bajo / obvio que no voy a repetir lo que me dijeron pero / el hombre que le iba en la pisadera me sacó a mi mamá(DIN) // así tal / ya me di claro // y yo bajé // no supe pero pa y ehperé poh porque también tenía que ehperar poh / ve que cuando vi ya que la liebre se perdió / que no bajó nadie / yo ahí me entré pa[ra] mi / pa[ra] la calle que yo vivía (SCHI_M32_067).

Cabe destacar que, en este caso, lo que nos interesa dejar de manifiesto es el aglutinamiento de ocurrencias de variantes específicas del DD en un segmento de discurso relativamente acotado. Este aglutinamiento y proliferación de casos de DD es más frecuente en narraciones construidas por mujeres que por hombres.

En el Gráfico 5, a continuación, se presenta la frecuencia de empleo del discurso referido de acuerdo con la edad de los informantes. 


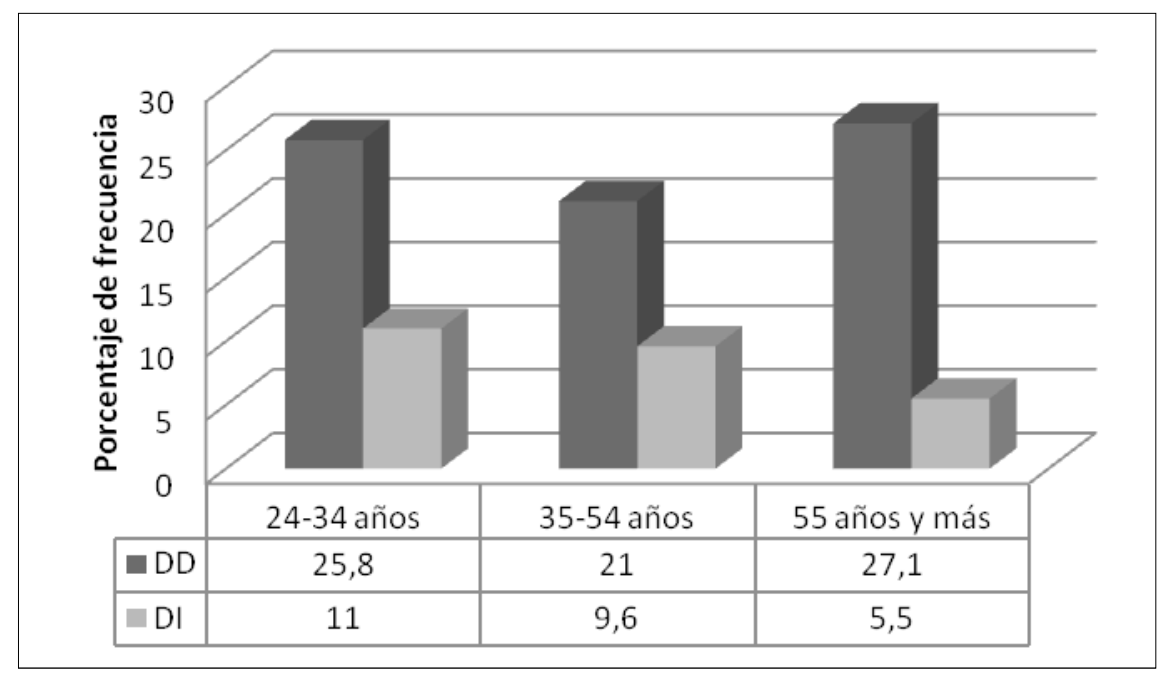

Gráfico 5. Variantes generales de discurso referido según edad.

Como se desprende de los datos del Gráfico 5, el empleo del DD se produce de manera similar entre el primer y el tercer grupo etario: con un 25,8\% (283/1.095) para el grupo más joven y con un 27,1\% (297/1.095) para los integrantes del tercer grupo de edad; sin embargo, la frecuencia de empleo del discurso referido disminuye levemente para el grupo de mediana edad, que registró un 21\% (230/1.095) de casos. Por lo que respecta al DI, en cambio, conforme aumenta la edad de los sujetos que conforman la muestra, se observa una disminución constante de dicha variante, esto es, el grupo de edad más joven manifestó una frecuencia del 11\% (120/1.095) de DI; luego, en el grupo de edad intermedia disminuye a un 9,6\% (105/1.095) y, finalmente, los sujetos de edad más avanzada, solo emplean el DI en un 5,5\% (60/1.095). Como consecuencia, la distancia entre el empleo del DD y el DI es mucho más ostensible en el grupo etario de 55 años y más.

Finalmente, en el Gráfico 6 se presenta el análisis del discurso referido según el nivel educacional de los informantes.

Tal como se observa en el Gráfico 6, al correlacionar el empleo del discurso referido con el nivel educacional de los informantes, el DD es el que se utiliza con mayor frecuencia en los tres niveles, aunque con una mayor tendencia en el nivel educacional medio con el 31,8\% (348/1.095), en tanto que su empleo disminuye en el nivel bajo con el 23\% (251/1.095) y desciende aún más en el nivel alto, que registró un 19,5\% (214/1.095) de las ocurrencias. El DI, por su parte, se mantiene relativamente constante en los dos primeros niveles, con un 7,9\% (87/1.095) y un 7,8\% (85/1.095), respectivamente, y aumenta su frecuencia mínimamente en el tercer nivel con el 10\% 
(110/1.095). Por consiguiente, mientras el empleo de DI se mantiene relativamente constante en los tres niveles educaciones, el DD manifiesta un 'patrón curvilíneo' en su comportamiento.

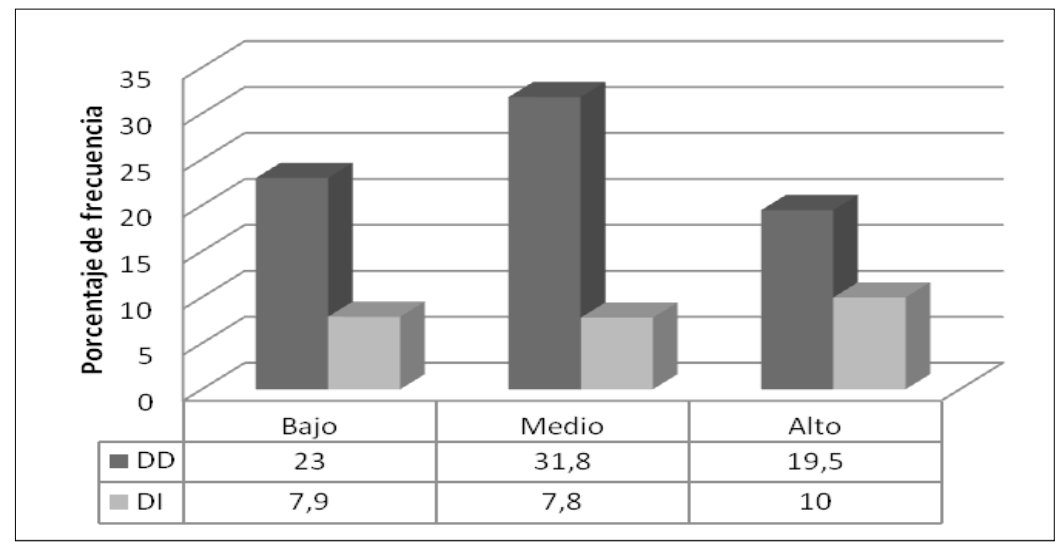

Gráfico 6. Variantes generales de discurso referido según nivel educacional.

\section{Discusión}

La preponderancia del empleo del DD por sobre DI ha sido confirmado también por Van der Houwen (2000) para el habla de la Ciudad de México, por Camargo (2008) en materiales del PRESEEA-Alcalá (España) y por Gallucci (2009) para el habla de Caracas (Venezuela). Llama la atención la escasa frecuencia del discurso directo con pronombre personal (DDPRO) verificada en nuestros materiales. Este último resultado contrasta con los de Cameron (1998) en su estudio sobre las formas de manifestación del estilo directo en San Juan (Puerto Rico), quien destaca esta variante como la más vernacular (Cameron, 1998). Probablemente, este contraste se deba a la tendencia a expresar el pronombre sujeto con mayor frecuencia sobre todo en el habla caribeña (Blas Arroyo, 2005).

Con respecto al menor empleo del DI en hablantes del tercer grupo etario (55 años y más), en nuestra opinión, una posible explicación radicaría en la mayor complejidad sintáctica de la mencionada variante y en los cambios en el rendimiento de habilidades lingüísticas derivadas del envejecimiento. Como ya señalamos, el empleo del DI implica una reformulación sintáctica, ya que las expresiones referenciales y el sistema deíctico deben adecuarse, de modo que el empleo del 'que' de complemento y la cláusula subordinada redundan en una estructura de mayor complejidad. Esto podría, eventualmente, relacionarse con los hallazgos de los estudios sobre los efectos del envejecimiento en la estructuración discursiva y sintáctica que, en general, han reportado efectos en la memoria, el lenguaje y las funciones ejecutivas (Véliz, 2004; Labos, Del Río \& Zavala, 2009). 
Por último, uno de nuestros hallazgos más relevantes es la alta frecuencia de aparición del DD en el discurso narrativo de las mujeres que integran la muestra. Hemos considerado que dicha variante podría interpretarse como una estrategia pragmática a través de la cual, las mujeres dotan de mayor teatralidad y dinamismo a sus historias, transformándolas en verdaderos 'mini-dramas', cuestión que ya había sido verificada en el habla de Santiago de Chile por Prieto y San Martín (2004-2005) y Guerrero (2011a). Asimismo, consideramos relevante señalar que las narraciones analizadas constituyen lo que Labov (1972) denomina "narración de experiencia personal", definida como:

"one method of recapitulating past experience by matching a verbal sequence of clauses to the sequence of events which (it is inferred) actually occurred [un método de recapitulación de la experiencia pasada adecuando una secuencia verbal de proposiciones a la secuencia de sucesos que (se supone) ocurrieron realmente (la traducción es nuestra)]" y que son, por tanto, historias "dignas de contarse" (Labov, 1972: 360).

La definición laboviana es fundamental, debido a que nos permite explicar la presencia de mecanismos lingüísticos que justifican la narración. Así, el discurso referido y, sobre todo, el DD podría ser uno de estos mecanismos lingüísticos que, de acuerdo con propuestas complementarias a la de Labov y Waletzky (1967) y Labov (1972), corresponde a un recurso evaluativo (Shiro, 1998; Jiménez, 2006), esto es, aquellos fragmentos que le permiten al narrador validar su narración o, en otras palabras, dejar en claro cuál es la razón de ser de su relato y su meta al narrarlo. En tanto recurso evaluativo, el discurso referido, en general, y el DD, en particular, tendría como uno de sus objetivos transformar la historia en un relato más 'entretenido', en términos de lo que hemos denominado 'mini-drama'.

\section{CONCLUSIONES}

En relación con el empleo de las variantes generales del discurso referido en el corpus PRESEEA-SA, relevamos un empleo preponderante de DD $(74,2 \%)$ en comparación con la frecuencia registrada de DI (25,8\%). Asimismo, el DD suele utilizarse en su variante específica más convencional (DDC), esto es, mediante el uso de un verbo de habla (verba dicendi) como introductor de la cita (51\%), en tanto que la variante específica sin dicho verbo introductor (DDL) presentó una frecuencia ostensiblemente menor $(21,5 \%)$, de igual modo que aquella en la que se utiliza un pronombre personal para introducir la cita (DDPRO) manifestó un empleo casi irrelevante en el corpus analizado (1,7\%). Por otra parte, en el caso del Discurso Indirecto, su variante específica convencional (DIC) en la que emplea el 'que' como complementizador fue la más empleada por los informantes $(16,1 \%)$, en comparación con la variante específica (DIN) en la que los sujetos hacen una referencia narrativizada a un acto discursivo anterior $(9,7 \%)$. 
Por lo que se refiere a la variable interna analizada, esto es, el tipo de secuencia discursiva (narrativa o argumentativa) en la que se utilizó el discurso referido, los integrantes de la muestra lo emplearon preferentemente en secuencias de tipo narrativo (61,8\% para el DD y 19,2\% para el DI), mientras que en las secuencias de tipo argumentativo, el empleo del discurso referido disminuyó de manera ostensible en el caso del DD (13\%) y se mantuvo aún más escaso en el DI (6\%).

Al correlacionar la frecuencia de empleo del discurso referido con las variables sociodemográficas de los sujetos bajo estudio, observamos que: a) hubo una frecuencia similar de empleo de las variantes generales de discurso referido según el sexo de los informantes, aunque se registró un mayor porcentaje de aparición en las mujeres con un $40,8 \%$ de DD y un $14,6 \%$ de DI, frente al $33,5 \%$ de DD y el $11,1 \%$ de DI en los hombres; b) en relación con el grupo de edad, el DD se manifiesta con porcentajes similares en el primer y tercer grupo etario (25,8\% y 27,1\%, respectivamente), mientras que en el segundo grupo de edad la frecuencia de DD baja, aunque no de manera ostensible (21\%); el empleo de DI, por su parte, disminuye conforme avanza la edad de los sujetos: 11\% (20-34 años), 9,6 (35-54 años) y 5,5 (55 años y más), posiblemente, debido a la mayor complejidad sintáctica que implica el empleo del DI, lo que determina que a medida que avanza la edad de los sujetos de la muestra se lo emplea en menor medida; por último, c) con respecto al nivel educacional de los informantes, concluimos que el DD presenta una mayor frecuencia en el nivel medio $(31,8 \%$ ) y disminuye en los niveles bajo (23\%) y alto (19,5\%), mientras que el empleo del DI se manifiesta de manera más o menos constante en los tres niveles educacionales: 7,9\% (bajo), 7,8\% (medio) y 10\% (alto).

En consideración a los resultados obtenidos en este estudio, podemos destacar, además, que existe una preponderancia en el empleo de DD en una misma intervención de modo aglutinado o condensado en el caso de las mujeres que integran la muestra, construyendo, de esta manera, una especie de 'mini-dramas' en sus relatos. Esta capacidad de incluir una gran cantidad de voces ajenas en el discurso propio de manera aglutinada no se observó en los relatos masculinos; es decir, serían las mujeres las que emplean este recurso pragmático como una estrategia que les permite 'teatralizar' y hacer más dinámicas sus historias. Este fenómeno ya había sido identificado en una muestra de hablantes representativos del estrato bajo del español de Santiago de Chile por Prieto y San Martín (2004-2005).

Finalmente, en esta investigación corroboramos la posibilidad de estudiar el discurso referido como un caso de variable sociolingüística en un sentido amplio del concepto, esto es, en el caso del discurso referido los hablantes tienen la opción de escoger alternativamente entre dos valores o variantes generales al momento de citar el discurso enunciado en otras situaciones de comunicación: el DD y el DI, cada una con variantes específicas. 


\section{REFERENCIAS BIBLIOGRÁFICAS}

Aijón Oliva, M. A. \& Serrano, M. J. (2010). Las bases cognitivas del estilo lingüístico. Sociolinguistic Studies, 4(1), 115-144.

Bajtín, M. (1985). Estética de la creación verbal. México: Siglo Veintiuno Editores.

Benveniste, E. (1970). L'appareil formel de l'énonciation. Langages, 17, 12-18.

Blas Arroyo, J. L. (2005). Sociolingüistica del español. Desarrollos y perspectivas en el estudio de la lengua española en contexto social. Madrid: Cátedra.

Camargo, L. (2004). El estilo directo y el estilo indirecto. De las gramáticas del español al análisis del discurso y la pragmática. En M. Villayandre Llamazares (Coord.), Actas del V Congreso de Lingüistica General (pp. 479-489). Madrid: Arco/Libros.

Camargo, L. (2006). El diálogo reconstruido y la imagen positiva. En M. Casado, R. González \& M. V. Romero Gualda (Eds.), Análisis del discurso: Lengua, cultura, valores (pp. 1241-1253). Madrid: Arco/Libros.

Camargo, L. (2008). La representación del discurso en los corpus PRESEEA: Metodología y propuesta de análisis. Ponencia presentada en el XV Congreso Internacional de la Asociación de Lingüística y Filología de América Latina (ALFAL), Montevideo, Uruguay.

Cameron, R. (1998). A variable syntax of speech, gesture, and sound effect: Direct quotations in Spanish. Language Variation and Change, 10, 43-83.

Chafe, W. (1979). Interpretation and involvement in spoken and written language. Ponencia presentada en of the Second World Congress of the International Association for Semiotic Studies, Vienna.

Chafe, W. (1980). The peer stories: Cognitive, cultural and linguistic aspects of narrative productions. Norwood, Nj: Ablex.

Chafe, W. (1982). Integration and involvement in speaking, writing and oral literature. En D. Tannen (Ed.), Spoken and written language. Exploring orality and literacy (pp. 35-53). Norwood, NJ: Ablex.

Coupland, N. (2007). Style: Language variation and identity. Cambridge: Cambridge, University Press.

Ducrot, O. (1986). El deciry lo dicho. Barcelona: Paidós.

Eckert, P. \& Rickford, J. (2001). Style and sociolinguistic variation. Cambridge: Cambridge University Press. 
Gallucci, M. J. (2009). Nos fuimos a la casa, y mi mamá: “¿Estaban lanzándose por la quebrada?”, “¡No, mamá!”, “Claro que sí!”: Estudio piloto de las citas conversacionales en el habla de Caracas. Núcleo, 26, 75-98.

García, E. (1986). El fenómeno (de)queísmo desde una perspectiva dinámica del uso de la lengua. En J. Moreno de Alba (Ed.), Actas del II Congreso Internacional sobre El español de América (pp. 46-65). México: Universidad Nacional Autónoma de México.

Genette, G. (1989). Figuras III. Madrid: Lumen.

Goffman, E. (1979). Footing. Semiótica, 25, 1-29.

Guerrero, S. (2011a). Diferencias de género en evaluaciones de narraciones de experiencia personales en el habla juvenil de Santiago de Chile. Una aproximación sociolingüística. Revista Signos. Estudios de Lingüística, 44(75), 1832.

Guerrero, S. (2011b). Análisis sociolingüístico de las diferencias de género en patrones narrativos de historia de experiencia personal en el habla juvenil de Santiago de Chile. Boletín de Filología, Tomo XLVI(2), 85-106.

Jiménez, T. (2006). La narración infantil. Un estudio en niños de educación básica. Revista de Investigación, 60, 157-174.

Kristeva, J. (1986). Word, dialogue, and the novel. En T. Moi (Ed.), The Kristeva Reader (pp. 35-61). Nueva York: Columbia University Press.

Labos, E., Del Río, M. \& Zabala, K. (2009). Perfil de desempeño lingüístico en el adulto mayor. Revista Argentina de Neuropsicologia, 13, 1-13.

Labov, W. (1972). Sociolinguistic patterns. Philadelphia: University of Pennsylvania Press.

Labov, W. (1978). Where does the sociolinguistics variable stop? A response to Beatriz Lavandera. Texas Working Papers in Sociolinguistics, 44, 1-17.

Labov, W. \& Waletzky, J. (1967). Narrative analysis. En J. Helm (Ed.), Essays on the verbal and visual arts (pp. 12-44). Seattle: University of Washington Press.

Lavandera, B. (1978). Where does the sociolinguistic variable stop? Language in Society, 7, 171-182.

Lenski, G. (1954). Status crystallization: A non-vertical dimension of social status. American Sociological Review, 19, 405-413.

Lenski, G. (1956). Social participation on status crystallization. American Sociological Review, 21, 458-464. 
López Morales, H. (2004a). Sociolingüística. Tercera edición aumentada. Madrid: Gredos.

López Morales, H. (2004b). La investigación sociolingüística en Hispanoamérica durante los últimos veinticinco años. Lingüistica Española Actual, XXVI(2), 151-173.

Maldonado, C. (1991). Discurso directo y discurso indirecto. Madrid: Taurus.

Maldonado, C. (1999). Discurso directo y discurso indirecto. En I. Bosque \& V. Demonte (Eds.), Gramática descriptiva de la lengua española (pp. 3549-3596). Madrid: Real Academia Española-Espasa Calpe.

Moreno Fernández, F. (1998). Principios de sociolingüisticay sociología del lenguaje. Barcelona: Ariel.

Perret, M. (1994). L'enonciation en grammaire du texte. París: Nathan.

Prieto Vera, L. (1995-1996). Análisis sociolingüístico del dequeísmo en el habla de Santiago de Chile. Boletín de Filologia, Tomo XXXV, 379-452.

Prieto, L. \& San Martín, A. (2002-2003). Diferencias de género en el empleo del discurso referido: Aproximación sociolingüística y pragmático-discursiva. Boletin de Filología, Tomo XXXIX, 269-304.

Reyes, G. (1984). Polifonía textual. Madrid: Gredos.

Reyes, G. (1990). La pragmática lingüistica. Barcelona: Montesinos.

Reyes, G. (1993). Los procedimientos de cita: Estilo directo y estilo indirecto. Madrid: Arco Libros.

San Martín, A. (2004-2005). Igual como marcador discursivo en el habla de Santiago de Chile: Función pragmático-discursiva y estratificación social de su empleo. Boletin de Filologia, Tomo XL, 201-232.

San Martín, A. (2011). Los marcadores interrogativos de control de contacto en el corpus PRESEEA de Santiago de Chile. Boletín de Filología, Tomo XLVI(2), 135-166.

Sankoff, D. (1988). Sociolingüística y variación Sintáctica. En F. Newrneyer (Ed.), Panorama de la lingüistica moderna (pp. 140-161). Madrid: Visor.

Searle, J. (1969). Speech acts. Cambridge: Cambridge University Press.

Serrano, M. J. (1999). Estudios de variación sintáctica. Madrid/Frankfurt: Iberoamericana/ Vervuert. 
Serrano, M. J. (2006). Gramática del discurso. Madrid: Akal.

Serrano, M. J. (2007). Historia que ya es historia: Evolución y actualidad del concepto y metodología de la variación sintáctica. Boletín de Lingüistica, 28, 102-127.

Serrano, M. J. (2011). Sociolingüistica. Barcelona: Ediciones del Serbal.

Shiro, M. (1998). Los pequeños cuentacuentos. El desarrollo de las habilidades narrativas en niños de edad escolar. Caracas: Universidad Central de Venezuela.

Silva-Corvalán, C. (2001). Sociolingüistica y pragmática del español. Washington, D.C.: Georgetown University Press.

Tannen, D. (1982). Oral and literate strategies in spoken and written narratives. Language, 58(1) 1-21.

Van der Houwen, F. (2000). El habla directa vs. indirecta y la organización del discurso. Foro Hispánico, 17, 27-40.

Véliz, M. (2004). Procesamiento de estructuras sintácticas complejas en adultos mayores y adultos jóvenes. Estudios Filológicos, 39, 65-81.

\section{NOTAS}

1 Dicha controversia ha estado en el centro de la discusión sociolingüística, especialmente, a partir de los estudios de variación sintáctica en el inglés, el francés de Montreal y el español, entre otras lenguas. Como es sabido, en el marco del paradigma cuantitativo laboviano más clásico, una exigencia para la aplicación de este concepto era que las formas alternantes no conllevaran diferencias semánticas y, de esta manera, consistieran en 'dos o más formas diferentes de decir lo mismo’ (Labov, 1972; Moreno Fernández, 1998; López Morales, 2004a, 2004b). No obstante, con posterioridad a este debate, se ha defendido la necesidad de relativizar la exigencia de igualdad semántica de las variantes y reemplazarla por un criterio de comparabilidad o equivalencia funcional o pragmática (Lavandera, 1978; Silva-Corvalán, 2001; Serrano, 2007, 2011), supuesto que está en el centro de la fundamentación de este trabajo.

2 Otras dimensiones del estudio del discurso referido en lengua española son abordadas en Cameron (1998) y Camargo $(2004,2006)$.

3 La extensión del concepto de variable sociolingüística al estudio de fenómenos de variación sintáctica, planteó una serie de dificultades, especialmente, a partir del trabajo de Lavandera (1978) en el que se cuestionó explícitamente dicha extensión arguyendo que no estaba garantizado en el estudio de dichas variables el principio de invariabilidad a nivel semántico, imprescindible para la consideración de una variable sociolingüística. Para mayor detalle en cuanto al desarrollo de esta controversia Lavandera (1978), Labov (1978), García (1986), Prieto (1995-1996), Sankoff (1988), Serrano (1999, 2007, 2011), Silva-Corvalán (2001), López Morales (2004) y Blas Arroyo (2005).

4 El equipo del PRESEEA en Santiago de Chile está compuesto por los profesores de la Universidad de Chile, Alfredo Matus y Luis Prieto (coordinadores) y Abelardo San Martín y Silvana Guerrero (investigadores). 
5 Dicho instrumento fue implementado por el profesor Dr. Luis Prieto Vera, quien estuvo a cargo de la mencionada asignatura entre 1978 y 2009.

6 Estudio Sociolingüístico del Español de Chile (ESECH) es un grupo de investigación de la Universidad de Chile al que pertenecen los miembros del Equipo PRESEEA-Santiago.

7 Según Lenski $(1954,1956)$, se considera que un individuo es congruente con su estatus cuando las puntuaciones obtenidas en las diferentes dimensiones usadas para medir el estatus, son más o menos iguales, independiente de que sus rangos sean altos, bajos o estén en una extensión media. Cuando las puntuaciones del individuo son muy diferentes, se habla de incongruencia de status.

8 Cabe mencionar que, para facilitar la comprensión de los casos de discurso referido, en esta sección, se utilizarán las siguientes convenciones:

- En la presentación de los ejemplos, se transcribirá cada uno en forma de cita incluyendo la cantidad de contexto necesaria para su comprensión e, indicando, al final de cada una, entre paréntesis, el código del sujeto que la emitió.

- Tanto para los comentarios analíticos como para los ejemplos, se utilizarán las mismas siglas que se emplearán en la matriz de análisis para identificar las distintas variantes específicas de discurso referido.

- Cada tipo general y específico de discurso referido será debidamente identificado en los ejemplos, con negrita si es discurso directo y con cursiva, si es discurso indirecto. Finalizado el segmento de discurso referido, se indicará, entre paréntesis, la sigla del tipo específico de discurso empleado.

Respecto de la transcripción de los ejemplos, es necesario señalar que: 1) se emplearon las siguientes convenciones para señalar a los participantes de las entrevistas del corpus: $\mathrm{E}=$ entrevistador e I = informante; 2) el texto correspondiente a cada ejemplo de las entrevistas se transcribe en ortografía convencional, incluidos los acentos gráficos. Las palabras que presentan elisiones en su pronunciación se completan en su escritura con paréntesis cuadrados. En el caso de las aspiraciones, estas se marcarán con h; 3) para la mejor comprensión de los ejemplos que hemos incluido en este artículo, se ha suprimido el sistema de etiquetas pertenecientes al Standard Generalized Markup Language (SGML) contenidas en el Text Encoding Initiative (TEI), que se emplean en la transcripción de las entrevistas pertenecientes al PRESEEA, excepto las pausas.

10 A este respecto, cabe señalar la precisión hecha por Tannen (1982) de que integración y fragmentación tienen que ver con rasgos superficiales de la estructura lingüística, mientras que implicación y distanciamiento se relacionan con una dimensión más profunda, que reflejaría lo que Goffman (1979) ha descrito como footing en la interacción cara a cara, término que indicaría la postura del emisor frente a su auditorio, y al que Tannen (1982) ampliaría su alcance para incluir, asimismo, la postura del emisor hacia el material o contenido de su discurso.

\section{* Agradecimientos}

En este artículo se presentan resultados parciales del Proyecto VID SOC 09/18-2, "Variación sintáctica y discursiva del español de Santiago de Chile", financiado por la Vicerrectoría de Investigación y Desarrollo de la Universidad de Chile. 